\title{
Inhibition of DNA Repair by Neighbouring Mismatched Bases in Streptococcus pneumoniae
}

\author{
By ANNE-MARIE GASC, PEDRO GARCIA† AND MICHEL SICARD* \\ Centre de Recherches de Biochimie et Génétique Cellulaires du CNRS, 118 route de Narbonne, \\ 31062 Toulouse Cedex, France
}

(Received 31 May 1988; revised 25 July 1988)

\begin{abstract}
A set of pneumococcal strains containing immediately adjacent or nearby double mutations at the amiA locus, conferring resistance to amethopterin, has been isolated by oligonucleotide sitespecific mutagenesis. Repair of these double mutations has been measured by transformation of wild-type strains with DNA extracted from these strains. In several transformations we have observed an inhibition of repair by neighbouring mismatches. This inhibition ranges from mild to severe depending upon the interfering mismatch. Unrepaired mismatches can strongly inhibit repair of an adjacent repairable mutation. This suggests that the repair-complex proteins attach not only to repairable mismatches but also to some mismatches known to escape the repair system.
\end{abstract}

\section{INTRODUCTION}

A central role for DNA polymerase in the fidelity of DNA replication has been documented in numerous studies (Drake et al., 1969; Ripley, 1981; Ripley \& Shoemaker, 1983). In vitro, using well-characterized multi-enzyme complexes, the errors in DNA replication are several orders of magnitude more frequent than spontaneous mutations observed in vivo. It has been suggested that a post-replication repair mechanism efficiently contributes to fidelity (Hibner \& Alberts, 1980) and that the most frequent errors of replication would be specifically eliminated by a correction process (Radman \& Wagner, 1986). The first evidence for such a correcting system was obtained in studies of spontaneous mutants at the amiA locus in Streptococcus pneumoniae. These mutants occur at high frequency because the locus is quite long and it contains several successive open reading frames (Gasc, 1987). The spontaneous mutation rate was found to be higher in a strain unable to repair mismatched bases at the amiA locus (Tiraby \& Sicard, 1973) as well as in other genes (Tiraby \& Fox, 1973). The spontaneous mutants which are efficiently eliminated by the wild-type repair system were eventually sequenced. The mutations were shown to be either transitions, or one- or two-base additions or deletions (Claverys et al., $1981 \mathrm{~b}$, 1983; Lacks et al., 1982; Gasc \& Sicard, 1986; Gasc et al., 1987). As the frequency of transitions and transversions remaining after repair is similar, it is likely that the repair system corrects the most common errors in DNA replication, i.e. transitions.

The mismatch repair system that eliminates some errors of replication also acts at the level of heteroduplex DNA during recombination, where it is more easily studied. Recombination between markers during transformation in S. pneumoniae depends strongly on the marker scored (Ephrussi-Taylor et al., 1965; Lacks, 1966). It is possible to correlate mutational changes in the DNA of each marker with its ability to be repaired (reviewed by Claverys \& Lacks, 1986): both $\mathrm{G} / \mathrm{T}$ and $\mathrm{C} / \mathrm{A}$ mismatches resulting from transitions are most prone to repair, as well as $\mathrm{G} / \mathrm{G}$ which results from a transversion; most $\mathrm{C} / \mathrm{T}$ mismatches are repaired; $\mathrm{A} / \mathrm{A}$ and $\mathrm{T} / \mathrm{T}$ are partially repaired; $\mathrm{A} / \mathrm{G}$ is poorly or not at all repaired; $\mathrm{C} / \mathrm{C}$ has never been found to be repaired.

† Present address: Centro de Investigaciones Biologicas (CSIC), Velasquez 144, 28006 Madrid, Spain. 
One- or two-base additions or deletions are most frequently repaired whereas longer heterologies (more than five base-pairs) completely escape this repair system (Gasc et al., 1987).

The repair mechanism excises several kilobases of DNA to eliminate a single mismatched base (Mejean \& Claverys, 1984). This accounts for the observation that a mismatch prone to repair can provoke the repair of a non-repairable mismatch according to the distance between them (Lacks, 1966; Sicard \& Ephrussi-Taylor, 1966; Sicard et al., 1985). However, when two mismatches are separated by very few base-pairs, we have observed some cases in which repair of both can be depressed (Claverys et al., 1983). Studies of this latter effect require construction of very closely linked markers that is quite difficult to achieve by classical genetic means but is feasible by site-directed mutagenesis.

In this paper we describe mutants obtained by this method. They contain nearby or adjacent double-base substitutions. We demonstrate that the double mutations create interferences in the process of excision and repair of mismatched bases, suggesting that the repair protein complex can attach to a non-repairable mismatch without effecting its repair.

\section{METHODS}

Bacterial strains and genetic analyses. The pneumococcal strains used in this study were derivatives of the R6 strain. Amethopterin-resistant mutants at the amiA locus (Sicard \& Ephrussi-Taylor, 1965) were isolated in strain R6 hex- 801 (Lefevre et al., 1979) and were resistant to a concentration of $10^{-5} \mathrm{M}$-amethopterin. Isolation and purification of DNA, and transformation procedures, were similar to those described by Claverys et al. (1981 a). Efficiency of transformation was measured in strain R6 hex +800 (Lefevre et al., 1979) as the ratio of amethopterinresistant transformants to streptomycin-resistant (str-4l) transformants.

Statistical analysis of efficiencies was performed by the method of Kimball (1961). Briefly, if $x$ and $y$ are the total number of colonies on each selective medium, and $Z$ is the ratio $x / y$, then the variance of $Z$ is

$$
V_{z}=\frac{x}{y^{3}}(x+y)
$$

This calculation assumes that $x$ and $y$ follow a Poisson distribution. This is a reasonable assumption since $x$ and $y$ are always larger than 100 and usually more than 500 .

Induction of amiA mutants by oligonucleotide-directed mutagenesis. Wild-type fragments A1 and B2 belonging to the ami $A$ locus (Gasc et al., 1987) were cloned in phage M13 derivatives (Zoller \& Smith, 1982). Single-stranded DNA was extracted and hybridized with synthetic oligonucleotides complementary to the wild-type pneumococcal sequence except for the bases to be substituted. The procedures described by Kramer et al. (1984) and by Norris et al. (1982) were used to obtain these mutants in Escherichia coli. They were sequenced and the M13 replicating form was transformed into strain $\mathrm{R} 6$ hex- 801 , deficient in mismatch repair. It was possible to induce these mutants directly in pneumococcus by another procedure, as follows. M13 DNA containing the cloned Al or B2 fragments was hybridized to the required oligonucleotide, and second-strand polymerization and ligation was carried out as described by Kramer et al. (1984). After linearization by $B g l \mathrm{II}$, samples were used to transform the wild-type pneumococcal strain 801 and amethopterin-resistant transformants were selected. Up to $2 \times 10^{5}$ mutants $\mathrm{ml}^{-1}$ were obtained. Unstable strains that resulted from rare insertion-duplication events were discarded. Stable mutants were intercrossed to ascertain their homoallelism. One transformant for each site-directed mutagenesis was sequenced.

\section{RESULTS}

Six double mutants in the A1 fragment and one in the B2 fragment of the amiA locus have been obtained by site-directed mutagenesis. All of them contain a non-sense mutation yielding the highest level of resistance to amethopterin. They consist either of adjacent base substitutions or of substitutions separated by one, two or three base-pairs. The base changes involve either transitions associated with several classes of transversions, similar transversions or a transversion with an A/T deletion (Table 1). Such a variety of double mismatched bases enables us to address the question: Is a repairable or an unrepairable mismatch able to interfere with the repair of a second and neighbouring mismatch?

Repair can be measured by transformation efficiency since the respective values are inversely related (Gray \& Ephrussi-Taylor, 1967). The results of these experiments are reported in Table 1 . The transformation efficiency of strain amiA-135 is 0.15 , the same as that found for fully 


\section{Table 1. Transformation efficiency of double mismatches}

Wild-type bacteria were transformed by DNA carrying a double mutation and the reference marker str-41. Transformation efficiency is the ratio of amethopterin transformants to streptomycin transformants. Without interference, the predicted transformation efficiency is the efficiency of the best-repaired single-site mutation: when both heteroduplexes are repaired this value is 0.15 on the average; when only one heteroduplex DNA is repaired this value is $1 \cdot 20$. Repair is inversely related to transformation efficiency. Sequences are written in the order $5^{\prime} \rightarrow 3^{\prime}$ corresponding to the mRNA strand. A1, wild-type sequence in the A1 fragment of the amiA locus in the amiA-36 region; B2, wildtype sequence in the B2 fragment of the amiA locus in the amiA-128 region (Gasc et al., 1987). Statistical fluctuations were calculated as described in Methods.

\begin{tabular}{|c|c|c|c|c|c|}
\hline Strains & Sequence & $\begin{array}{c}\text { Mismatched } \\
\text { heteroduplexes }\end{array}$ & $\begin{array}{c}10^{-5} \times \text { No. of } \\
\text { amiA trans- } \\
\text { formants } \mathrm{ml}^{-1}\end{array}$ & $\begin{array}{c}10^{-6} \times \text { No. of } \\
\text { str- } 2 \text { trans- } \\
\text { formants ml-1 }\end{array}$ & $\begin{array}{l}\text { Transfor- } \\
\text { mation } \\
\text { efficiency }\end{array}$ \\
\hline$a m i A^{+}(\mathrm{A} 1)$ & CCAGATTCAT & & & & \\
\hline amiA-135 & $-------\mathrm{AG}-$ & $\frac{\mathrm{CA}}{\mathrm{T}}$ and $\frac{\mathrm{GT}}{\mathrm{CA} G}$ & $\begin{array}{l}4 \cdot 89 \\
3 \cdot 82\end{array}$ & $\begin{array}{l}3 \cdot 20 \\
2 \cdot 73\end{array}$ & $\begin{array}{l}0.15 \pm 0.02 \\
0.14 \pm 0.03\end{array}$ \\
\hline amiA-142 & $----\mathbf{G}--\mathbf{A}--$ & $\frac{\mathrm{A}--\mathrm{C}}{\mathrm{C}--\mathrm{T}}$ and $\frac{\mathrm{T}--\mathrm{G}}{\mathrm{G}--\mathrm{A}}$ & $\begin{array}{l}1 \cdot 75 \\
1 \cdot 46\end{array}$ & $\begin{array}{l}7 \cdot 11 \\
5 \cdot 20\end{array}$ & $\begin{array}{l}0.25 \pm 0.03 \\
0.28 \pm 0.04\end{array}$ \\
\hline$a m i A-148$ & $-----\mathrm{C}-\mathrm{A}--$ & $\frac{T-C}{G-T}$ and $\frac{A-G}{C-A}$ & $\begin{array}{l}2 \cdot 02 \\
1 \cdot 32\end{array}$ & $\begin{array}{l}7 \cdot 33 \\
5 \cdot 51\end{array}$ & $\begin{array}{l}0.27 \pm 0.03 \\
0.24 \pm 0.03\end{array}$ \\
\hline amiA- 145 & $-------\mathrm{A}-\mathrm{C}$ & $\frac{C-T}{T-G}$ and $\frac{G-A}{A-C}$ & $\begin{array}{l}1.08 \\
0 \cdot 82\end{array}$ & $\begin{array}{l}3 \cdot 62 \\
3 \cdot 15\end{array}$ & $\begin{array}{l}0.30 \pm 0.04 \\
0.26 \pm 0.03\end{array}$ \\
\hline$a m i A-136$ & TG $\cdots \cdots-\cdots$ & $\frac{\mathrm{CC}}{\mathrm{AC}}$ and $\frac{\mathrm{GG}}{\mathrm{TG}}$ & $\begin{array}{l}29 \cdot 8 \\
13 \cdot 4\end{array}$ & $\begin{array}{l}4 \cdot 30 \\
1 \cdot 81\end{array}$ & $\begin{array}{l}0.69 \pm 0.09 \\
0.74 \pm 0.07\end{array}$ \\
\hline amiA-146 & $-----\mathrm{G}-\mathrm{A}--$ & $\frac{\mathrm{T}-\mathrm{C}}{\mathrm{C}-}$ and $\frac{\mathrm{A}-\mathrm{G}}{\mathrm{TG}-\mathrm{A}}$ & $\begin{array}{l}67 \cdot 2 \\
23 \cdot 5\end{array}$ & $\begin{array}{l}5 \cdot 60 \\
2 \cdot 20\end{array}$ & $\begin{array}{l}1 \cdot 20 \pm 0 \cdot 12 \\
1.07 \pm 0 \cdot 10\end{array}$ \\
\hline $\operatorname{amiA}^{+}(\mathrm{B} 2)$ & CGTTTTAATG & & & & \\
\hline amiA-126 & $--\mathrm{TTTTT}--\mathrm{T}$ & $\frac{\mathrm{T}---\mathrm{G}}{\mathrm{AA}--\mathrm{A}}$ and $\frac{\mathrm{A}-\cdots \mathrm{C}}{\mathrm{TT}--\mathrm{T}}$ & $\begin{array}{l}3.69 \\
1.49\end{array}$ & $\begin{array}{l}1 \cdot 15 \\
4 \cdot 95\end{array}$ & $\begin{array}{l}0.32 \pm 0.04 \\
0.30 \pm 0.05\end{array}$ \\
\hline
\end{tabular}

repaired single mismatches (Gasc \& Sicard, 1986). One of the substitutions in this strain is a transition which is always repaired. Therefore the $A / G$ mismatch which is not repairable does not interfere with the repair of adjacent $G / T$ and $C / T$ mismatches and does not modify the repair of $\mathrm{A} / \mathrm{C}$. This absence of interference between highly and poorly repairable markers when they are adjacent is similar to the same observation when they are far apart (Lacks, 1966; Gray \& Ephrussi-Taylor, 1967; Sicard et al., 1985).

An interference was found, however, when strain amiA-142 was used in transformation. In this strain, the same mismatches as in amiA-135 are separated by two base-pairs. The efficiency of transformation was twice the value expected if there was no interference. Therefore at least one of the three mismatches $\mathrm{A} / \mathrm{C}, \mathrm{T} / \mathrm{G}, \mathrm{C} / \mathrm{T}$, which are individually repairable, partially escapes correction due to the presence of another mismatch located two base-pairs away.

A similar interference was found for the two strains amiA-148 and amiA-145. The base substitutions here result from two transitions, one of them being the same transition, $\mathrm{C} / \mathrm{G} \rightarrow \mathrm{A} / \mathrm{T}$, as in strain amiA-142. A similar result was also obtained when a fully repairable mutation (addition of $A / T$ ) was present three bases away from the mismatches $A / G$ and $C / T$, in strain amiA-126.

The inhibition reported above was small but reproducible. It became much larger in strain amiA-136, where the efficiency of transformation was increased fourfold over that obtained with fully repaired mutations (Table 1). In this case, a mismatch derived from a transition is adjacent to a $\mathrm{C} / \mathrm{C}$ or a $\mathrm{G} / \mathrm{G}$ mismatch. At least one heteroduplex is poorly repaired. It was possible to determine which heteroduplex is repaired because the elimination of a mismatch extends over several kilobases and will depress transformation efficiency if the excluded strands are opposite. This depression is a function of the distance between the markers (Sicard \& Ephrussi-Taylor, 1966). We have used strain $a m i A-9$ rev- 81 , which results from a $\mathrm{C} / \mathrm{G} \rightarrow \mathrm{G} / \mathrm{C}$ transversion. In a 
cross between this strain and wild-type DNA, the conserved mismatch is $C / C$ whereas $G / G$ is fully repaired. When amiA-9 rev-81 was transformed by amiA-136 str-4I DNA, the efficiency of transformation was $0 \cdot 38$. This is much lower than the level of non-repaired mismatches $(1 \cdot 2)$. This depression, which is not reduced to the basic level $(0 \cdot 15)$ due to recombination between the markers, cannot result from the elimination of the $\mathrm{G} / \mathrm{G}$ mismatch at site amiA-9, which is anyway fully repaired. Therefore the conserved double mismatch is $\mathrm{AC} / \mathrm{CC}$ in the pairing between wild-type and amiA-136. The mismatch $\mathrm{C} / \mathrm{C}$, which alone is unrepaired, strongly inhibits repair of the adjacent $\mathrm{A} / \mathrm{C}$ mismatch. This is an agreement with a previous observation at another site (Claverys et al., 1983).

A question that one might ask is whether the close association of two identical mismatches known to be poorly repaired would induce some repair. Pairing between amiA-146 and wild-type involves the A-G/G-A double mismatches which are not repaired when alone. Results indicate that this structure is not repaired because the transformation efficiency of amiA-146 by wildtype DNA is 1.20 , i.e. the same efficiency as observed when only one of these mismatches is present (Table 1). Moreover, using the same methodology as for strain amiA-136, we have found that the only repaired mismatches are $\mathrm{T}-\mathrm{C} / \mathrm{C}-\mathrm{T}$. For this double mutant there was no interference.

\section{DISCUSSION}

The main results reported in this work show that some unrepairable mismatches are able to inhibit repair at a nearby site. This effect can be small or much larger depending upon the mutations. It thus seems possible that in double mutants not only the nature of the mismatches but the neighbouring sequences may affect repair, as has been reported for some single-site mutations (see Claverys \& Lacks, 1986, for review). Moreover, as for single-site mutations (Modrich, 1987), it is impossible to attribute any physico-chemical state of the DNA to account for these interferences.

Although the concept and the first evidence for excision and repair of mismatched bases were reported long ago in pneumococcus by Ephrussi-Taylor \& Gray (1966) to account for the elimination of single-site mutations during recombination, the mechanism of repair is only partially understood. From the facts that long deletions are not repaired by this system (Ephrussi-Taylor et al., 1965; Lacks, 1966) and that the most systematically repaired mismatches are transition mutations, it was obvious that this process can accommodate only a very limited distortion of the DNA helix. This concept was further substantiated by the demonstration that the efficiency of repair is inversely proportional to the size of the heterology, as if looped-out structures could occur that escape recognition by the repair system (Gasc \& Sicard, 1986). In fact, nuclear magnetic resonance studies of oligonucleotides have shown that a transition mismatch is more often intra-helical than an unrepaired transversion mismatch (Fazakerley et al., 1986), supporting the role of DNA structure for repair specificity of mismatched bases. Extra-helical structures are more likely to occur when more than two mutations are clustered. This could explain why ami $A-29$, a spontaneous mutant, is not repaired (Ephrussi-Taylor et al., 1965). This strain contains three adjacent mutations, one of them being the addition of a base-pair (Claverys et al., 1981 b) that by itself would be repaired.

However, DNA structures alone cannot explain some differences in repair efficiency between pneumococcus and $E$. coli or other organisms like yeast. In pneumococcus, $\mathrm{C} / \mathrm{T}$ is repaired most of the time, and the complementary mismatch $\mathrm{A} / \mathrm{G}$ is not repaired, whereas in $E$. coli these two mismatches are both repaired at the same level. A difference between pneumococcus and yeast is that in the latter organism $\mathrm{A} / \mathrm{A}$ and $\mathrm{T} / \mathrm{T}$ are repaired at the same level as transitions, which in turn are less repaired than single-base insertions (White et al., 1985; Jones et al., 1987). It would appear, therefore, that proteins recognizing repairable mismatches differ slightly in their specificity from one organism to another. Their affinity for some mismatched bases must depend upon neighbouring sequences in order to account for differences in repair efficiency between identical transversions located at several sites in the same organism.

To explain the reported inhibition of repair by an unrepairable nearby mismatch we propose that one or several proteins of the repair complex are able to bind not only to the repairable 
mismatches but also to others, especially $\mathrm{C} / \mathrm{C}$. This binding would inhibit attachment of this complex to nearby mismatches that are individually repairable. This model is supported by the demonstration that purified mutS protein, the $E$. coli homologue to the pneumococcal hexA protein of the repair complex (Claverys \& Lacks, 1986), binds not only to repairable mismatches but equally well to unrepairable ones (Su \& Modrich, 1986). The repaired mismatches are presumably further processed by an exonuclease activity on donor DNA by the hex system. This complex would travel along the DNA molecule and would degrade the free ends of donor DNA before its ligation to the chromosomal DNA as proposed by Guild \& Shoemaker (1976). Such processing would not occur for unrepairable single-site mismatches like $\mathrm{C} / \mathrm{C}$ that may hold onto the protein complex. One could expect then that competition for the repair complex occurs between neighbouring mismatches. However, there is no direct biochemical evidence in support of this hypothesis since the products of the pneumococcal hex genes have not been purified. The specificity of the systems that repair mismatched bases at the replication or the recombination levels would result from the dual effect of DNA secondary structure and the recognition by the protein complex at repairable mismatches.

We thank B. Stevens for help in preparing the manuscript. P. Garcia was supported by a fellowship from the Fundacion Juan March.

\section{REFERENCES}

Claverys, J. P. \& LaCKs, S. (1986). Heteroduplex deoxyribonucleic acid base mismatch repair in bacteria. Microbiological Reviews 50, 133-165.

Claverys, J. P., Louarn, J. M. \& Sicard, A. M. (1981a). Cloning of Streptococcus pneumoniae DNA: its use in pneumococcal transformation and in studies of mismatch repair. Gene $13,65-75$.

Claverys, J. P., Mejean, V., Gasc, A. M., Galibert, F. \& SICARD, A. M. $(1981 b)$. Base specificity of mismatch repair in Streptococcus pneumoniae. $\mathrm{Nu}$ cleic Acids Research 9, 2267-2280.

Claverys, J. P., Mejean, V., Gasc, A. M. \& Sicard, A. M. (1983). Mismatch repair in Streptococcus pneumoniae: relationship between base mismatches and transformation efficiencies. Proceedings of the National Academy of Sciences of the United States of America 80, 5956-5960.

Drake, J. W., Allen, E. F., Forsberg, S. A., Preparata, R. M. \& Greening, E. O. (1969). Genetic control of mutation rates in bacteriophage T4. Nature, London 221, 1128-1132.

EPHRUSSI-TAYLOR, H. \& Gray, T. C. (1966). Genetic studies of recombining DNA in pneumococcal transformation. Journal of General Physiology 49, 211-231.

EPhrussi-Taylor, H., Sicard, A. M. \& Kamen, R. (1965). The problem of relative efficiency of transforming factors. Genetics 51, 455-475.

Fazakerley, G. V., Quignard, E., Woisard, A., Guschlbauer, W., Van Der Marel, G. A., Van BOOM, J. H., Jones, M. \& Radman, M. (1986). Structure of mismatched base-pairs in DNA and their recognition by the Escherichia coli mismatch repair system. EMBO Journal 5, 3697-3703.

GaSC, A. M. (1987). Etude de la spécificité du système de séparation des mesappariements au cours de la transformation chez Streptococcus pneumoniae. Thèse es Sciences, University Paul Sabatier of Toulouse.

Gasc, A. M. \& Sicard, A. M. (1986). Frameshift mutants induced by quinacrine are recognized by the mismatch repair in Streptococcus pneumoniae. Molecular and General Genetics 203, 269-273.

Gasc, A. M., Garcia, P., Baty, D. \& Sicard, A. M. (1987). Mismatch repair during pneumococcal transformation of small deletions produced by sitedirected mutagenesis. Molecular and General Genetics 210, 369-372.

Gray, T. C. \& EPHRUSSI-TAYLOR, H. (1967). Genetic recombination in DNA-induced transformation of pneumococcus. V. The absence of interference, and evidence for the selective elimination of certain donor sites from the final recombinants. Genetics 57, 125-153.

Guild, W. R. \& ShoemakeR, N. B. (1976). Mismatch correction in pneumococcal transformation: donor length and hex-dependent marker efficiency. Journal of Bacteriology 125, 125-135.

HibneR, U. \& AlberTs, M. (1980). Fidelity of DNA replication catalysed in vitro on a natural DNA template by the $\mathrm{T} 4$ bacteriophage multi-enzyme complex. Nature, London, 285, 300-305.

JONES, M., WAGNER, R. \& RADMAN, M. (1987). Repair of a mismatch is influenced by the base composition of the surrounding nucleotide sequence. Genetics $115,605-610$.

Kimball, A. W. (1961). Confidence intervals for recombination experiments with microorganisms. Biometrics 17, 150-153.

Kramer, W., Drutsa, V., hansen, H. W., Kramer, B., Pflugfelder, M. \& Fritz, H. J. (1984). The gapped duplex DNA approach to oligonucleotide directed mutation construction. Nucleic Acids Research 12, 9441-9456.

LACKS, S. (1966). Integration efficiency and genetic recombination in pneumococcal transformation. Genetics 53, 207-235.

LaCKs, S., DunN, J. \& Greenberg, B. (1982). Identification of base mismatches recognized by the heteroduplex-DNA-repair system of Streptococcus pneumoniae. Cell 31, 327-336. 
Lefevre, J. C., Claverys, J. P. \& Sicard, A. M. (1979). Donor deoxyribonucleic acid length and marker effect in pneumococcal transformation. Journal of Bacteriology 138, 80-86.

Mejean, V. \& Claverys, J. P. (1984). Effect of mismatched base-pairs on the fate of donor DNA in transformation of Streptococcus pneumoniae. Molecular and General Genetics 197, 467-471.

MODRICH, P. (1987). DNA mismatch correction. Annual Review of Biochemistry 56, 435-466.

Norris, K., Norris, F., Christiansen, L. \& Fill, N. (1982). Efficient site-directed mutagenesis by simultaneous use of two primers. Nucleic Acids Research 11, 5103-5112.

RADMAN, M. \& WAGNER, R. (1986). Mismatch repair in Escherichiacoli. Annual Reviewof Genetics 20, 523-538.

RIPLEY, L. S. (1981). Influence of diverse gene 43 DNA polymerases on the incorporation and replication in vivo of 2-aminopurine at A.T base-pairs in bacteriophage T4. Journal of Molecular Biology 150, 197-216.

RIPLEY, L. S. \& SHOEMAKER, N. B. (1983). A major role for bacteriophage T4 DNA polymerase in frameshift mutagenesis. Genetics 103, 353-366.

SICARD, A. M. \& EPHRUSSI-TAYLOR, H. (1965). Genetic recombination in DNA-induced transformation of pneumococcus. II. Mapping the amiA region. Genetics 52, 1207-1227.

Sicard, A. M. \& EpHrussi-TaYlor, H. (1966). Recombinaison génétique dans la transformation chez le pneumocoque. Etude des reversions au locus amiA. Comptes rendus de l'Académie des sciences 262 , 2305-2308.

Sicard, A. M., Lefevre, J. C., Mostachfi, P., Gasc, A. M., MéJean, V. \& Claver ys, J. P. (1985). Longand short-patch conversions in Streptococcus pneumoniae transformation. Biochimie 67, 377-384.

SU, S. \& MODRICH, P. (1986). Escherichia coli mutS protein binds to mismatched DNA base-pairs. Proceedings of the National Academy of Sciences of the United States of America 83, 5057-5061.

TIRABY, G. \& Fox, M. S. (1973). Marker discrimination in transformation and mutation of pneumococcus. Proceedings of the National Academy of Sciences of the United States of America 70, 3541-3545.

TIRABY, G. \& SiCARD, A. M. (1973). Integration efficiencies of spontaneous mutant alleles of amiA locus in pneumococcal transformation. Journal of Bacteriology 116, 1130-1135.

White, J. H., Lusnak, K. \& Fogel, S. (1985). Mismatch-specific post-meiotic segregation frequency in yeast suggests a heteroduplex recombination intermediate. Nature, London 315, 350352.

ZolleR, M. J. \& SMITH, M. (1982). Oligonucleotidedirected mutagenesis using M13 derived vectors : an efficient and general procedure for the production of point mutations in any fragment of DNA. Nucleic Acids Research 10, 6487-6500. 